\title{
VINCULACÃO EM MÃES ADOLESCENTES E SEUS BEBÉS
}

\author{
Ines Jongenelen ${ }^{1}$ \\ Isabel Soares ${ }^{2}$ \\ Karin Grossmann ${ }^{3}$ \\ Carla Martins $^{4}$
}

Resumo: Neste artigo, as autoras apresentam uma investigação empírica com mães adolescentes e seus bebés, conduzida sob a perspectiva da Teoria da Vinculação de Bowlby.

Quarenta adolescentes e seus bebés foram avaliados na gravidez e $12 .^{\circ}$ mês do pós-parto, com base, respectivamente, na Adult Attachment Interview e na Situação Estranha. Os resultados revelam que a maioria dos bebés apresenta uma organização de vinculação segura à mãe, aos 12 meses de idade. Não foi encontrada uma associação significativa entre a classificação das mães na $A A I$ e a classificação dos seus bebés na Situação Estranha, quer ao nível dos três padrões, quer em função da dimensão segurança versus insegurança da vinculação.

Palavras-chave: vinculação, maternidade na adolescência

Attachment organization in adolescent mothers and their infants (Abstract): Within Bowlby's attachment theory framework, an empirical research with forty adolescent mothers and their infants assessed during pregnancy and at $12^{\text {th }}$ months postpartum is presented. Our results show no statistically significant pattern of intergenerational transmission of attachment from mother to infant, and a substantial number of infants classified as secure to the mother at 12 months of age.

Key-words: attachment, adolescent motherhood

\footnotetext{
${ }^{1}$ Professora do Instituto Superior Dom Afonso III. Correspondência sobre este artigo deverá ser enviada para o e-mail ijongenelen@hotmail.com.

2 Professora catedrática do Departamento de Psicologia, Instituto de Educação e Psicologia, Universidade do Minho.

${ }^{3}$ Investigadora aposentada da Universidade de Regensburg, Alemanha.

4 Professora auxiliar do Departamento de Psicologia, Instituto de Educação e Psicologia, Universidade do Minho.
}

PSICOLOGIA, Vol. XX (1), 2006, Edições Colibri, Lisboa, pp. 11-36 


\section{Introdução}

Se os estudos de Ainsworth e as pesquisas longitudinais subsequentes evidenciaram o papel das relações nas diferenças individuais na organização da vinculação e no desenvolvimento ao longo da infância, a identificação, por Main e colaboradores, de padrões de organização mental da vinculação em adultos, análogos aos padrões de organização comportamental na infância, permitiu a exploração empírica da transmissão intergeracional da vinculação, que Bowlby (1979) tinha sublinhado ao considerar que a qualidade da vinculação é transmitida ao longo das gerações, não pelos genes, mas pela microcultura familiar.

No estudo pioneiro de Main e colaboradores (1985), no âmbito de uma investigação longitudinal, foi encontrada uma concordância significativa entre a organização da figura de vinculação na $A A I$ e a organização comportamental de vinculação do bebé com essa figura na Situação Estranha. O Quadro 1 apresenta de forma esquemática as organizações de vinculação obtidas a partir da Adult Attachment Interview (AAI) e os padrões correspondentes de organização comportamental da vinculação avaliados com a Situação Estranha.

Em 1995, na meta-análise de van IJzendoorn, baseada em estudos conduzidos em diversos países, foi encontrada uma correspondência de $75 \%$ entre a segurança da vinculação da figura parental (avaliada através da $A A I)$ e a segurança da relação de vinculação entre a figura parental e o bebé (avaliada através da Situação Estranha ou por procedimentos de avaliação similares). $O$ efeito preditor da qualidade da vinculação da figura parental na qualidade de vinculação do(a) filho(a) foi destacado. A partir daí, correspondências relevantes têm sido documentadas em vários estudos, quer sejam prospectivos, retrospectivos ou concorrentes, utilizando a AAI e a Situação Estranha (Ammaniti, Speranza \& Candelori, 1996; Benoit \& Parker, 1994; Gloger-Tipplet \& Gomille, 1999; Pederson, Gleason, Moran \& Bento, 1998; Radojevic, 1994; Soares, 1996; Steele, Steele \& Fonagy, 1996) também com mães adolescentes (Carlson, 1990; Levine \& Tuber, 1991; Ward \& Carlson, 1995).

Apesar de vários estudos publicados apontarem no sentido de uma concordância intergeracional entre a organização da vinculação da figura parental e do(a) filho(a), importa enfatizar que esta concordância não é absoluta. Há díades que não evidenciam concordância e há estudos que não apresentam resultados significativos. De facto, em cerca de $25 \%$ das famílias, não se verifica uma correspondência entre a segurança de vinculação da figura parental e do bebé (cf. van IJzendoorn, 1996). Além disso, como destaca Soares (2002) 
Quadro 1: Correspondência entre as classificações da vinculação na Adult Attachment Interview e na Situação Estranha

\section{Adult Attachment Interview}

Seguro/Autónomo (F). Discurso coerente e colaborativo mantido durante a descrição e avaliação das experiências relacionadas com a vinculação, quer estas sejam descritas como favoráveis ou desfavoráveis. $\mathrm{O}$ sujeito parece valorizar a vinculação, sendo objectivo no que diz respeito a cada experiência ou relação em particular.

Desligado (D). Não coerente. Descrições positivas e normalizadoras das figuras parentais que não são apoiadas ou são contraditas por memórias específicas. As experiências negativas são desvalorizadas quanto ao seu efeito no desenvolvimento. As entrevistas são curtas, muitas vezes com insistência na falta de memória.

Preocupado (E). Não coerente. Preocupado com as experiências, parecendo zangado, confuso e passivo, ou com medo e sobrecarregado. As entrevistas são longas, com algumas respostas irrelevantes e declarações vagas ou emaranhadas.

Não resolvido/Desorganizado $(U)$. $\mathrm{Na}$ discussão de temas de perdas ou abuso, revelam lapsos na monitorização do raciocínio ou discurso; por exemplo, pode referir-se à pessoa morta como se ainda estivesse fisicamente viva, pode permanecer em silêncio ou usar um discurso laudatório. Pode complementar a classificação das categorias D, F ou E.

\section{Situação Estranha}

Seguro (B). Mostra sinais de sentir a falta da figura parental durante a primeira separação e chora durante a segunda separação. Saúda activamente a figura parental, procurando a proximidade e $\mathrm{o}$ contacto. Após ter mantido o contacto com a figura parental, acalma-se e volta à exploração. Revela uma óbvia preferência pela figura parental face à Estranha.

Evitante $(A)$. Não chora durante a separação, focalizando-se nos brinquedos e no ambiente durante todo o procedimento. Evita e ignora activamente a figura parental na reunião, por exemplo, afastando-se, virando as costas, ou rejeitando, quando é pegado ao colo. As respostas à figura parental parecem não emocionais.

Ambivalente/Resistente (C). Preocupado com a figura parental durante o procedimento; pode parecer zangado ou passivo. Durante a reunião, revela dificuldade $\mathrm{em}$ se acalmar e em voltar à exploração, continuando a centrar-se na figura parental e a chorar.

Desorganizado/Desorientado (D).

Exibe comportamentos desorganizados e desorientados na presença da figura parental, sugerindo um colapso temporário da estratégia comportamental; e.g., pode ficar imobilizado com uma expressão de transe, com as mãos no ar; pode levantar-se e depois deixar-se cair com a entrada da figura parental; ou agarra-se enquanto se inclina para trás. Pode complementar a classificação das categorias $\mathrm{A}, \mathrm{B}$ ou $\mathrm{C}$.

Nota: Adaptado de Main (1996) e de Hesse (1999). 
"esta concordância intergeracional, tal como a qualidade da vinculação não
é imutável: mudanças normativas e acontecimentos de vida críticos poderão
ser geradores de novas dinâmicas relacionais e de reorganizações do com-
portamento e do pensamento sobre a vinculação, no sentido adaptativo ou
inadaptativo" (p. 58).

No âmbito da maternidade na adolescência, as questões da vinculação assumem contornos específicos. Na perspectiva da vinculação, a adolescência é o tempo de aprendizagem do "tornar-se uma figura de", mas um tempo em que "ser uma figura vinculada" é ainda vital. É neste cruzamento do "vir a ser" e do "ser" que se inscreve o núcleo da vinculação na adolescência (Soares, 2001).

A maternidade na adolescência poderá ser concebida, em geral, como um acontecimento de vida não normativo que vai obrigar a uma reorganização pessoal e relacional que garanta novos modos de expressão e de realização, adequados à nova situação (Jongenelen, 1998). Assim, podemos pensar que o que vai acontecer à jovem que engravida e tem um filho é que vai viver uma transição de papéis acelerada: a adolescente é ainda receptora de cuidados (por parte das suas figuras parentais), ao mesmo tempo que se torna numa figura de vinculação para o filho (Jongenelen, 2004).

\section{Método}

\section{Participantes}

Participaram neste estudo quarenta adolescentes e seus bebés. No último trimestre de gravidez a idade das adolescentes variava entre 13 e 18 anos $(M=16,55, D P=1,32)$, tendo sido uma gravidez não planeada para a maioria destas jovens, que também se enquadra num nível socioeconómico baixo (90\% NSE baixo, 10\% NSE médio), vivendo em zonas urbanas ou suburbanas. No último trimestre de gravidez, 52,5\% das adolescentes estavam casadas ou viviam com o companheiro. Quanto ao estatuto escolar e profissional, $75 \%$ das adolescentes tinha completado menos de nove anos de escolaridade e mais de metade das participantes, durante a gravidez, não estudava nem trabalhava ( $20 \%$ estudavam e $27,5 \%$ eram trabalhadoras). Em relação à organização do contexto familiar no $12 .^{\circ}$ mês do pós-parto, $37,5 \%$ das adolescentes residiam apenas com o seu cônjuge ou companheiro $20 \%$ apenas com a família nuclear, $22,5 \%$ com ambos, uma minoria com o pai do bebé e respectiva família (5\%) e $12,5 \%$ das adolescentes com este e outros familiares. 
Os bebés apresentavam a distribuição equitativa quanto ao sexo e a maioria $(75,0 \%)$ nasceu de uma gestação de termo. Aos 12 meses de idade, para $57,5 \%$ dos casos, a mãe partilhava a prestação de cuidados maioritariamente com a avó do bebé.

\section{Instrumentos de avaliação}

\section{Adult Atachment Interview}

A Adult Atachment Interview-AAI (George, Kaplan \& Main, 1985, versão portuguesa de Soares, 1992) foi utilizada para avaliar a representação da vinculação das grávidas adolescentes. Trata-se de uma entrevista semiestruturada, biográfica, de tipo clínico, organizada em torno de um conjunto de temas que traduzem as principais questões da vinculação, de acordo com a teoria de Bowlby (1969-1984, 1973, 1980).

A entrevista inicia-se com um breve resumo da história familiar, estimulando-se o sujeito a recordar as suas relações com os pais na infância, através da escolha de cinco adjectivos para caracterizar cada uma dessas relações. Progressivamente, são abordadas situações específicas e críticas do ponto de vista da relação de vinculação, nomeadamente, problemas ou dificuldades pessoais, doenças, acidentes, separações, situações de rejeição, ameaças e abandono pelas figuras de vinculação, castigos, maus tratos e perdas de figuras significativas. A entrevista contempla ainda questões de natureza mais geral e avaliativa, através das quais o sujeito é solicitado a analisar a influência das relações com os pais na infância, ao nível do seu desenvolvimento pessoal, as razões para o comportamento dos pais, bem como as mudanças ocorridas na relação ao longo dos anos. O sujeito é ainda questionado sobre as suas relações com outros adultos significativos e, na fase final da entrevista, sobre a sua relação actual com os pais. A abordagem de cada temática da entrevista é sempre efectuada a um nível mais geral (semântico) e a um nível mais específico (episódico). Deste modo, em cada questão, o sujeito é incentivado a recordar episódios ou acontecimentos ilustrativos das situações mencionadas e a avaliar o impacto dessas experiências no desenvolvimento da sua personalidade e das suas relações de vinculação.

A $A A I$ foi concebida de modo a possibilitar a activação do sistema de vinculação, levando o sujeito a evocar a história das suas relações com os pais, a partir das suas memórias e percepções. Neste âmbito, a entrevista tem como objectivo avaliar a representação actual do sujeito em relação à vinculação, focalizando-se no modo como as experiências e os seus efeitos são reflectidos, avaliados e integrados pelo indivíduo e traduzidos em termos de coerência narrativa e discursiva, ao longo da entrevista. Neste sentido, a tarefa central colocada ao sujeito é (1) produzir e reflectir acerca das 
memórias relacionadas com a vinculação e, simultaneamente, (2) manter um discurso coerente com o entrevistador (Hesse, 1999).

As entrevistas foram audiogravadas e transcritas e o método de análise e classificação da $A A I$ utilizado no nosso estudo empírico foi o Adult Attachment Q-sort, de Roger Kobak (Kobak, 1993; Kobak, Cole, Ferenz-Gillies, Fleming \& Gamble, 1993). Este método baseia-se no sistema de classificação da $A A I$ de Main e Goldwyn (1984-98), e consiste num conjunto de 100 itens $(Q-S e t)$ que avaliam os modelos internos dinâmicos dos participantes relativos ao self, à mãe e ao pai, bem como o processamento de informação e a regulação de emoções relativas à vinculação (Cole-Detke \& Kobak, 1996; Kobak et al., 1993; Kobak, 1993).

Um grupo de seis juízes, com treino prévio na utilização do referido método e cegos às classificações das entrevistas efectuadas pelos outros juízes, bem como face a outras medidas do estudo, trabalharam em pares e cotaram, de forma independente, cada transcrição. $\mathrm{O}$ acordo médio inter-juízes para a totalidade das entrevistas foi de 0,60 , variando entre 0,45 e 0,79 .

\section{Situação Estranha}

A Situação Estranha (Ainsworth \& Wittig, 1969; Ainsworth \& Bell, 1970; Ainsworth, Blehar, Waters \& Wall, 1978) foi utilizada com o objectivo de avaliar a organização comportamental da vinculação dos bebés participantes com a mãe.

A Situação Estranha é um procedimento laboratorial estandardizado, com a duração de 20 minutos e que envolve uma sequência fixa de oito episódios, que activam progressivamente o sistema de vinculação: a entrada num ambiente não familiar, a chegada de uma pessoa estranha, duas breves separações da figura de vinculação e duas subsequentes reuniões com ela (Ainsworth, et al., 1978). A análise dos comportamentos interactivos e da actividade exploratória do bebé, no decurso dos episódios da $\mathrm{Si}$ tuação Estranha, possibilita a classificação dos bebés num dos três grupos definidos por Ainsworth e colaboradores (1978): Padrão A - inseguro-evitante; Padrão B - seguro; e Padrão C - inseguro-resistente ou ambivalente; e, dentro de cada um destes, num determinado subgrupo.

Constituindo o método mais utilizado no quadro da pesquisa da vinculação na primeira infância, a Situação Estranha tem a sua validade e fidelidade bem estabelecida, ao longo de inúmeros estudos que investigaram aspectos vários da emergência de diferenças individuais no processo do desenvolvimento sócio-emocional.

No nosso estudo empírico, a totalidade dos bebés foi observada no procedimento laboratorial da Situação Estranha, o qual foi videogravado e posteriormente os vídeos foram analisados. Os 40 vídeos foram distribuí- 
dos aleatoriamente para classificação por um grupo de cinco juízes, com treino prévio na classificação da Situação Estranha ${ }^{5}$, segundo o sistema de Ainsworth e colaboradores (1978). Os juízes, cegos às classificações das entrevistas da vinculação das mães e a todas as outras medidas do estudo, trabalharam em pares e de forma independente classificaram cada vídeo. Assim, através da análise das observações videogravadas, os bebés foram classificados de acordo com quatro escalas interactivas: proximidade e procura de contacto; manutenção do contacto; resistência e evitamento para os Episódios 5 e 8 da Situação Estranha. Posteriormente, foram classificados de acordo com os grupos e subgrupos da vinculação descritos por Ainsworth e colaboradores (1978). Quando a classificação em termos de padrões $\mathrm{A}, \mathrm{B}$ e $\mathrm{C}$ não era concordante para o par de juízes, um terceiro juiz efectuou uma outra classificação do registo vídeo, com total desconhecimento dos resultados prévios obtidos pelos outros juízes. Um terceiro juiz foi necessário em $7(17,5 \%)$ dos casos.

No nosso estudo, o acordo inter-observadores na classificação dos padrões A, B e C atingiu o valor de $100 \%$ e na classificação dos subgrupos da vinculação atingiu o valor de $75 \%$, o que está dentro dos parâmetros internacionais considerados aceitáveis para o acordo de observadores treinados por Ainsworth ou pelos seus colaboradores.

\section{Procedimentos}

O presente estudo longitudinal teve como primeiro momento de avaliação o último trimestre de gravidez. A selecção da amostra obedeceu a dois critérios principais: primiparidade e idade igual ou inferior a 18 anos à data do parto. As participantes foram sequencialmente recrutadas em quatro unidades hospitalares do distrito do Porto, à medida que compareciam às consultas hospitalares, obedecendo aos critérios de selecção ante-

\footnotetext{
${ }^{5}$ A utilização do procedimento laboratorial da Situação Estranha exige que os investigadores tenham sido certificados para o efeito por um outro grupo de investigadores-formadores, credenciados por Mary Ainsworth. Tal certificação implica a realização de um programa de formação na Situação Estranha e a obtenção de níveis significativos de acordo inter-observadores, na cotação das escalas e na classificação dos padrões de vinculação, de acordo com o sistema de Ainsworth e colaboradores (1978), tendo em conta os critérios internacionalmente reconhecidos. A equipa de análise dos vídeos da Situação Estranha desta publicação integra o Grupo de Estudos da Vinculação (GEV), coordenado por Isabel Soares (Universidade do Minho), e obteve formação e certificado de fiabilidade na "Classificação da Qualidade da Vinculação na Situação Estranha" de acordo com o "Sistema de Ainsworth", sob orientação científica de Karin Grossmann da Universidade de Regensburg (Alemanha), entre 1993 e 1995. O certificado de fiabilidade implicou a classificação de 30 casos provenientes dos estudos conduzidos em Regensburg e Bielefeld (Alemanha).
} 
riormente mencionados. Numa primeira fase, no final de cada consulta, o médico informava a grávida adolescente do estudo em curso nesse hospital e convidava-a a participar. Numa segunda fase, que correspondeu ao nosso contacto inicial com as grávidas, os objectivos gerais do estudo foram explicados pela entrevistadora às adolescentes e a colaboração voluntária foi solicitada. A totalidade das grávidas adolescentes e o seu encarregado de educação (quando a adolescente era menor de idade) assinaram uma declaração de consentimento de participação. Neste primeiro momento de avaliação, recolheram-se dados de caracterização socio-demográfica das participantes e a Adult Attachment Interview foi administrada em contexto hospitalar.

Entre o $11 .^{\circ}$ e o $13 .^{\circ}$ mês de vida do bebé, as mães adolescentes e os bebés foram avaliados em contexto laboratorial no procedimento da Situação Estranha. As condições óptimas de avaliação foram asseguradas, dado que previamente se questionava a mãe acerca do estado de saúde do bebé, se estava com sono ou fome, o que levou, em alguns casos, a uma nova marcação das observações.

\section{Resultados}

Organização da vinculação das grávidas adolescentes

O Quadro 2 apresenta a distribuição das grávidas adolescentes segundo os padrões de vinculação e consequente representação (segura ou insegura) da vinculação.

Quadro 2: Classificação das grávidas adolescentes em termos de padrões de vinculação

\begin{tabular}{cccc}
\hline & Segura & Desligada & Preocupada \\
$\mathbf{N}(\%)$ & $17(42,5)$ & $16(40,0)$ & $7(17,5)$ \\
& Segura & & Insegura \\
& $17(42,5)$ & & $23(57,5)$ \\
\hline
\end{tabular}

Pela análise do Quadro 2 verifica-se que 42,5\% $(\mathrm{N}=17)$ das grávidas adolescentes foram classificadas no padrão autónomo-seguro em relação à vinculação, $40 \%(\mathrm{~N}=16)$ no padrão desligado/rejeitante e $17,5 \%$ $(\mathrm{N}=7)$ no padrão emaranhado/preocupado. Se analisarmos a distribuição das adolescentes em termos da dimensão segurança versus insegurança da representação da vinculação, conclui-se que 57,5\% $(\mathrm{N}=23)$ das grávidas adolescentes foram classificadas como inseguras e $42,5 \%(\mathrm{~N}=17)$ como seguras. 


\section{Organização da vinculação dos bebés}

Os Quadros 3 e 4 apresentam a distribuição dos bebés da amostra pelos diferentes padrões e subpadrões da vinculação, a partir da sua classificação na Situação Estranha (Ainsworth \& Wittig, 1969).

Quadro 3: Classificação dos bebés em termos de padrões de vinculação

\begin{tabular}{cccc}
\hline & Seguro (B) & Inseguro-Evitante (A) & Inseguro-Resistente (C) \\
\multirow{2}{*}{$\mathbf{N}(\%)$} & $23(57,5)$ & $13(32,5)$ & $4(10,0)$ \\
\cline { 2 - 4 } & Seguro & Inseguro \\
& $23(57,5)$ & $17(42,5)$ \\
\hline
\end{tabular}

Através da análise do Quadro 3 observa-se que 57,5\% dos bebés foram classificados como seguros e que, do grupo de bebés inseguros, 32,5\% foram classificados como inseguros-evitantes e $10 \%$ como inseguros ambivalentes/resistentes.

Quadro 4: Classificação dos bebés em termos de subpadrões de vinculação

\begin{tabular}{ccccccccc}
\hline N (\%) & A1 & A2 & B1 & B2 & B3 & B4 & C1 & C2 \\
& $8(20,0)$ & $5(12,5)$ & $7(17,5)$ & $8(20,0)$ & $4(10,0)$ & $4(10,0)$ & $2(5,0)$ & $2(5,0)$ \\
\hline
\end{tabular}

Atendendo à distribuição em termos de subpadrões da vinculação, como se pode observar no Quadro 4, entre os bebés inseguros-evitantes, o subpadrão com maior representatividade foi o $\mathrm{A} 1$, enquanto que entre os bebés seguros foi o subpadrão B2. Os quatro bebés inseguros-ambivalentes/resistentes distribuíram-se equitativamente pelos dois sub-padrões.

\section{Transgeracionalidade da vinculação}

O Quadro 5 apresenta a concordância entre a representação da vinculação das adolescentes, avaliada durante a gravidez, através da Adult Attachment Interview, e a organização comportamental da vinculação dos seus filhos, avaliados na Situação Estranha, aos 12 meses de idade. 
Quadro 5: Associação entre a classificação das grávidas adolescentes na $A A I$ e a classificação dos bebés na Situação Estranha, aos 12 meses de idade

\begin{tabular}{|c|c|c|c|}
\hline \multirow[b]{2}{*}{ Situação Estranha } & \multicolumn{3}{|c|}{$A \boldsymbol{A I}$} \\
\hline & $\begin{array}{c}\text { Segura (S) } \\
(\mathrm{N}=17)\end{array}$ & $\begin{array}{c}\text { Desligada (D) } \\
(\mathrm{N}=16)\end{array}$ & $\begin{array}{c}\text { Preocupada (P) } \\
\qquad(\mathrm{N}=7)\end{array}$ \\
\hline Seguro (B) $(N=23)$ & $11(9,8)$ & $8(9,2)$ & $4(4,0)$ \\
\hline Inseguro-Evitante (A) $(\mathrm{N}=13)$ & $6(5,5)$ & $6(5,2)$ & $1(2,3)$ \\
\hline Inseguro-Resistente $(\mathrm{C})(\mathrm{N}=4)$ & $0(1,7)$ & $2(1,6)$ & $2(0,7)$ \\
\hline $\mathrm{S} / \mathrm{D} / \mathbf{P} \rightarrow \mathrm{B} / \mathrm{A} / \mathrm{C}$ & & \multicolumn{2}{|c|}{ Segura/Insegura $\rightarrow$ Seguro/Insegurc } \\
\hline$\chi^{2}(4)=5,40$, n.s. & & \multicolumn{2}{|c|}{$\chi^{2}(1)=0,63$, n.s. } \\
\hline $\mathrm{k}=0,14$, n.s. & & \multicolumn{2}{|c|}{$\mathrm{k}=0,12, \mathrm{n} . \mathrm{s}$} \\
\hline
\end{tabular}

Nota: Os valores entre parêntesis representam frequências esperadas.

Pela análise do Quadro 5 constata-se não existir uma associação significativa entre a classificação das adolescentes na $A A I$ e a classificação dos seus bebés na Situação Estranha, quer ao nível dos três padrões, quer em função da dimensão segurança versus insegurança da vinculação.

Em termos dos três padrões, observou-se uma correspondência em $47,5 \%$ dos casos $(\mathrm{N}=19)$ (versus $39,3 \%, \mathrm{~N}=15,7$ de correspondência esperada), o que levou a que não fosse encontrada uma associação significativa entre as variáveis $\left(\chi^{2}(4)=5,40\right.$, n.s.; kappa $=0,14$, n.s.). Em termos de segurança versus insegurança, houve apenas 22 casos, representando $55,0 \%$ da amostra, onde se observou uma correspondência entre as classificações das mães e bebés (versus 49,0\%, $\mathrm{N}=19,6$ de correspondência esperada). Uma vez mais, não foi encontrada qualquer associação entre as duas variáveis $\left(\chi^{2}(1)=0,63\right.$, n.s.; kappa $=0,12$, n.s. $)$.

\section{Discussão}

\section{Organização da vinculação das mães adolescentes}

No nosso estudo, avaliámos a qualidade da organização da vinculação em mães adolescentes, maioritariamente enquadradas no nível socio-económico baixo, com base na Adult Attachment Interview. Os resultados revelaram que $42,5 \%$ das adolescentes avaliadas no último trimestre de gravidez foram consideradas com uma organização de vinculação segura e 
$57,5 \%$ com uma organização insegura, das quais $40 \%$ no padrão Desligado e $17,5 \%$ no padrão Preocupado/Emaranhado. Deste modo, encontrámos uma preponderância de mães adolescentes com uma organização de vinculação insegura.

Em primeiro lugar, comparativamente à distribuição média encontrada para mães adultas de amostras não clínicas (cf. meta-análise de van IJzendoorn \& Bakermans-Kranenburg, 1996), que aponta para $58 \%$ das mães consideradas como seguras, verificámos que a proporção de mães adolescentes com uma organização segura, obtida no nosso estudo, é inferior $(42,5 \%)$. Comparando a distribuição em termos de três padrões no nosso estudo com essa distribuição, verifica-se que é ao nível do padrão seguro e desligado que os resultados são mais dissimilares $(40 \%$ de desligadas versus $24 \%$ nas amostras não clínicas), já que a proporção de adolescentes preocupadas é semelhante (17,5\% no nosso estudo versus $18 \%$ nessa distribuição). No entanto, saliente-se que mesmo nessas amostras normativas se verifica variabilidade nas distribuições, já que a proporção de mães com uma organização de vinculação segura varia (entre $21 \%$ e $80 \%$, cf. van IJzendoorn \& Bakermans-Kranenburg, 1996). Numa análise mais detalhada, verificámos que a proporção de mães seguras em alguns desses estudos é semelhante à encontrada na nossa pesquisa, nomeadamente Main e Goldwyn (1998) encontraram 41\% de mães seguras, Crowell e Feldman (1988) 45\% e Zeanah e colaboradores (1993) 42\%.

Em segundo lugar, a preponderância de adolescentes com uma organização de vinculação insegura encontrada aproxima-se à da distribuição obtida em pesquisas com mães de nível socioeconómico baixo (cf. van IJzendoorn \& Bakermans-Kranenburg, 1996) ${ }^{6}$.

Embora, no nosso estudo, em termos do sistema de classificação de três categorias, $42,5 \%$ das adolescentes fossem avaliadas como seguras, das adolescentes inseguras, $40 \%$ foram enquadradas no padrão desligado em relação à vinculação. Tem sido sugerido que condições de vida sociais e económicas adversas poderão relegar a reflexão sobre as experiências relacionadas com a vinculação (característica dos sujeitos autónomos em relação à vinculação) para uma prioridade menor, explicando a sobre-representação de sujeitos desligados (van IJzendoorn \& Bakermans-Kranenburg, 1996). Nesta linha, Carlson (1990), discutindo os resultados da sua pesquisa com mães adolescentes, advoga mesmo que, de acordo com a noção de adaptação evolutiva de Bowlby (1969/1984), estratégias

\footnotetext{
${ }^{6}$ Neste tipo de populações, tende a verificar-se uma sobre-representação das categorias Desligado e "Não resolvido em relação à vinculação", esta última não avaliada na nossa pesquisa, dado a metodologia adoptada para a análise e classificação das entrevistas da vinculação - o método de $Q$-sort - não nos possibilitar a inclusão dos sujeitos nesta categoria.
} 
moderadamente defensivas poderão servir uma função adaptativa em contextos ambientais de risco, com múltiplos factores de stress económicos, familiares e sociais. Para além desta questão mais intimamente relacionada com o contexto socioeconómico de inserção destas mães, há que ter presente que, na nossa pesquisa, se tratava de uma amostra de adolescentes.

Assim, quando comparados com os resultados obtidos com pesquisas com adolescentes e jovens adultos de ambos os sexos sem filhos, e de amostras não clínicas (cf. meta-análise de van IJzendoorn \& Bakermans-Kranenburg, 1996; Allen, Hauser \& Borman-Spurell, 1996; Fonagy et al., 1996; Soares, 1996), no nosso estudo verifica-se uma proporção inferior de adolescentes seguras, uma superior de desligadas e, uma vez mais, uma proporção semelhante de adolescentes preocupadas. No panorama nacional, os nossos resultados não são concordantes com os obtidos por Soares (1996) numa pesquisa pioneira sobre a organização de vinculação na adolescência, na qual a maioria dos adolescentes $(78 \%)$ foram avaliados com uma organização segura. No entanto, destaque-se que, contrariamente ao nosso estudo, tratava-se de uma amostra de adolescentes de nível socioeconómico médio e elevado, na totalidade estudantes e solteiros, residindo com ambos os pais.

Por outro lado, destaque-se que, nos estudos anteriormente referidos (baixo risco), a idade dos sujeitos era, em média, superior à do nosso estudo (16,5 anos e onde $20 \%$ das adolescentes tinha menos de 16 anos), abarcando também, em algumas pesquisas, jovens universitárias. Sabemos que uma das tarefas de desenvolvimento da adolescência é a construção da autonomia, passando pela transformação da relação do(a) adolescente com as figuras parentais (Pipp et al., 1985). Poderemos, assim, colocar a hipótese que, sobretudo para as adolescentes mais novas, a nova dinâmica relacional entre necessidades de vinculação e de exploração e autonomia, a par das transformações de natureza cognitiva e emocional, que se estão a operar nesta fase do ciclo de vida, poderão levar a que a discussão das experiências com as figuras de vinculação na infância surja embebida de um certo distanciamento ou desvalorização das experiências de vinculação, característica dos sujeitos desligados (Main \& Goldwyn, 1984-1998). Como alertaram van IJzendoorn e Bakermans-Kranenburg (1996), ao discutir os resultados obtidos com adolescentes, o grupo dos mais jovens poderá revelar distribuições mais divergentes, já que, "no início da adolescência, a luta pela independência poderá ser mais intensa" (p. 18). Por outro lado, há igualmente que ter presente que, para estas adolescentes, a discussão, no decurso da entrevista, de um "passado" (i.e., a infância) relativo às experiências com as figuras de vinculação pode ser considerado ainda temporalmente recente, em comparação com adolescentes mais velhas ou, mesmo, sujeitos adultos. Como afirma Soares (1996a), a propósito do 
posicionamento dos adolescentes na $A A I$, "os adolescentes discursam sobre um sistema relacional que é ainda "ao vivo", no qual se move e envolve no quotidiano com as figuras parentais" (p. 302).

No entanto, são ainda relativamente escassas as pesquisas que utilizam a Adult Attachment Interview com adolescentes mais novos. No estudo longitudinal de Regensburg e Bielefeld na Alemanha, com uma amostra de 22 adolescentes de nível socioeconómico médio, aos 16 anos de idade, $50 \%$ foram classificados como seguros e $50 \%$ como inseguros (Zimmermann, Fremmer-Bombik, Spangler \& Grossmann, 1997), valores que são mais semelhantes aos obtidos por nós. Em nossa opinião, são necessárias mais pesquisas com adolescentes mais novos e de realidades socioeconómicas e culturais diversas, para que detenhamos um quadro mais claro da distribuição destes sujeitos pelos diferentes padrões de vinculação.

Em seguida, passaremos a um nível de discussão mais específico, comparando os resultados relativos à organização de vinculação das adolescentes avaliadas na gravidez, com os das escassas pesquisas com mães adolescentes.

Os nossos resultados são concordantes com os obtidos por Ward e Carlson (1995), também com uma amostra de adolescentes grávidas (média de 16.5 anos de idade, similar à nossa), embora com uma proporção ligeiramente superior de adolescentes com uma organização insegura (61\%). Quando nos focalizamos apenas no grupo das inseguras, o padrão de resultados é um pouco diferente: encontrámos um menor número de adolescentes no padrão desligado ( $40 \%$ versus $51 \%$ em Ward e Carlson, 1995) e um maior número no padrão preocupado/emaranhado (17,5\% versus $10 \%$ em Ward e Carlson, 1995), no sistema de três categorias. O facto de as adolescentes do estudo de Ward e Carlson (1995) estarem inseridas num contexto socioeconómico mais adverso do que o das adolescentes da nossa pesquisa - pertencerem a comunidades minoritárias urbanas da zona de Nova Iorque, caracterizadas por elevados índices de violência, e serem quase na totalidade solteiras - poderá contribuir para a proporção mais elevada de adolescentes desligadas, como tivemos oportunidade de discutir anteriormente.

Os nossos resultados são igualmente concordantes com os obtidos por Carlson (1990), numa amostra norte-americana, onde $45 \%$ das mães adolescentes foram avaliadas com uma organização de vinculação segura quando os filhos tinham 18 meses de idade, assim como numa amostra portuguesa (Matos, 2000), com características socioeconómicas e familiares similares às nossas ${ }^{7}$ e também avaliada no último trimestre de gravidez

\footnotetext{
${ }^{7}$ É de salientar que cerca de 50\% da amostra de Matos (2000) é comum à nossa amostra de adolescentes.
} 
com base no método de $Q$-sort, na qual $45 \%$ de adolescentes foram classificadas como seguras, $37,5 \%$ no padrão desligado e $17,5 \%$ no padrão preocupado.

Em síntese, a proporção elevada de mães adolescentes inseridas no padrão desligado parece-nos exigir a consideração quer do nível socioeconómico destas adolescentes - nível socioeconómico baixo - quer da sua idade - com uma média de 16 anos e com $20 \%$ de adolescentes com menos de 16 anos. Um marco fundamental, que ocorre na adolescência comparativamente à infância, é a emergência de uma organização de vinculação singular e integrada (Allen \& Land, 1999) e, nesse sentido, é importante compreender quando é que, no decurso da adolescência, a organização de vinculação se torna uma propriedade interna estável do indivíduo em desenvolvimento e não apenas o reflexo da relação actual com a figura de vinculação. Neste âmbito, poderemos pensar que talvez algumas das mães adolescentes mais jovens (13 ou 14 anos de idade), não tendo ainda integrado as experiências precoces de vinculação, poderão apresentar estratégias de distanciamento no decurso da entrevista face a tais experiências e, como tal, serem classificadas no padrão desligado, podendo, algum tempo mais tarde, vir a ser classificadas no padrão seguro. Para além disso, pelo facto de estarmos em presença de adolescentes provenientes de situações socioeconómicas desfavorecidas, consideramos pertinente o comentário de Weinfield e colaboradores (2000) que, discutindo as questões da descomtinuidade na organização intra-individual verificada ao longo do desenvolvimento, consideram que alguns indivíduos desligados, provenientes de situações socioeconómicas desfavorecidas, "might transition to earned security later if, as they mature, they are able to explore the meaning of their negative experiences" (2000, p. 701).

\section{Organização da vinculação dos bebés}

Os resultados relativos à organização comportamental da vinculação dos bebés, avaliados aos 12 meses de idade com a mãe no procedimento da Situação Estranha, revelaram que 57,5\% dos bebés foram classificados no padrão B - seguro. Tais resultados são convergentes com os obtidos em estudos, realizados em diversos países, com amostras pertencentes a populações de baixo risco que apontam para percentagens entre 50 a $75 \%$ de bebés seguros (cf. meta-análise de van IJzendoorn \& Kroonenberg, 1988). Estes resultados são, igualmente, concordantes com os obtidos em Portugal, nomeadamente por Soares e colaboradores (1995) e por Silva (1998, $60 \%$ ), embora no estudo de Soares e colaboradores (1995), com díades mãe-bebé de nível socioeconómico médio e mães adultas, a proporção de bebés seguros fosse superior $(69 \%)$. 
Os nossos resultados não são concordantes com os obtidos em amostras consideradas de risco, onde são incluídos, entre outros factores, $o$ nível socioeconómico baixo e a idade jovem da mãe e onde, em geral, se verificam percentagens inferiores de bebés com uma organização de vinculação segura (Weinfeld et al., 1999; van IJzendoorn, 1992).

Relativamente aos bebés inseguros, $32,5 \%$ foram considerados como evitantes e $10 \%$ como ambivalentes/resistentes. Embora a proporção de bebés evitantes seja um pouco superior à distribuição média dos estudos em diversos países (15 a 25\%, cf. van IJzendoorn \& Kroonenberg, 1988), parece corresponder a uma tendência patente nas nações da Europa Ocidental, onde a proporção de bebés inseguros-evitantes é, em geral, superior à encontrada em amostras dos Estados Unidos da América, Japão ou Israel (van IJzendoorn \& Kroonenberg, 1988; Grossmann et al., 1988; Soares et al., 1995; Silva, 1998). Destaque-se que, no âmbito nacional, a proporção de bebés inseguros-evitantes no nosso estudo é similar à encontrada por Silva (1998), junto de 48 díades mãe-bebé de nível socioeconómico médio, com 31,3\% de bebés inseridos nesse padrão de vinculação.

Comparando os nossos resultados, ao nível da organização da vinculação dos bebés, com outros estudos realizados com filhos de mães adolescentes, encontrámos alguma discrepância. Algumas pesquisas evidenciam proporções inferiores de bebés seguros, com valores que variam entre 24\% e 49\% (Ward \& Carlson; 1995; Levine \& Tuber, 1991; Whitman et al., 2001; Landy et al., 1983; Lamb et al., 1987; Rellinger, 1995; Broussard, 1995; Spencer, 1997). Em outras investigações, é semelhante a proporção de bebés seguros no nosso estudo, por exemplo, nas investigações de Carlson (1990), Frodi e colaboradores (1984, 1990), Hann e colaboradores (1988) e mais, recentemente, de Andreozzi e colaboradores (2002), que obtiveram, respectivamente, $50 \%, 53 \%, 68 \%$ e $66,7 \%$ de bebés inseridos no padrão B. A proporção de bebés inseguros-evitantes, embora mais elevada do que nos estudos com amostras normativas, em geral, é também concordante com a obtida em pesquisas com filhos de mães adolescentes (e.g. Carlson, 1990; Frodi et al., 1984; 1990; Levine \& Tuber, 1991; Ward \& Carlson, 1995).

A proporção de bebés seguros no nosso estudo, próxima da encontrada com mães adultas, é dissonante da visão, muitas vezes presente na literatura da maternidade na adolescência, que tende a enfatizar, apenas, os défices das mães adolescentes e seus filhos. Este resultado sugere que, mesmo face a situações socioeconómicas desfavorecidas e à idade jovem das mães - características que, no âmbito da literatura e investigação empírica da vinculação na infância, levam a que estas amostras sejam consideradas de risco - um elevado número de bebés é capaz de formar uma relação de vinculação segura com as suas mães. Os nossos resultados sugerem 
que muitas das mães adolescentes se constituem como base segura para estes bebés explorarem o mundo com confiança. No entanto, dado que, em termos da estrutura e da organização do contexto familiar, muitas destas díades residiram, ao longo do primeiro ano de vida, com outras figuras que, regularmente e numa base quotidiana, prestam cuidados ao bebé, nomeadamente as avós, não poderemos negligenciar o papel que estas figuras poderão ter na qualidade da vinculação do bebé à mãe. Em termos futuros, consideramos que mais pesquisas, com amostras de mães adolescentes e seus filhos, deverão explorar, em profundidade, o impacto da prestação de cuidados ao bebé pelas avós, na qualidade da vinculação do bebé à mãe. Será igualmente interessante explorar o impacto da própria representação da vinculação da avó, na qualidade da vinculação do bebé à mãe. Saliente-se que, embora no presente não detenhamos resultados sobre esta questão, no âmbito do projecto longitudinal em que a nossa pesquisa de insere, as avós maternas foram avaliadas com base na Adult Attachment Interview. Esperamos, num futuro breve, com a inclusão das três gerações - avó, mãe, bebé - alargar a nossa compreensão sobre as histórias da transgeracionalidade na maternidade adolescente. Por fim, e dada a saliência, nestes grupos, da prestação de cuidados pelas avós aos bebés, que parece ocorrer, seria interessante avaliar-se também a qualidade da vinculação dos bebés às avós. Pensando nas consequências a longo prazo para estes bebés seguros, no âmbito da investigação longitudinal da vinculação, existe evidência empírica de que a vinculação segura tende a estar associada a um desenvolvimento subsequente mais adaptativo (Weinfield et al., 1999; Thompson, 1999).

No entanto, apesar de a maioria dos bebés ter sido considerada segura, 42,5\% exibiram uma organização comportamental de vinculação insegura. Existe evidência empírica considerável de que as relações de vinculação inseguras na infância têm repercussões mais negativas a longo prazo, ao nível do desenvolvimento social e emocional do indivíduo, ao longo da sua trajectória de desenvolvimento, em comparação com a vinculação segura (Weinfield et al., 1999; Thompson, 1999), por exemplo, a organização de vinculação insegura na infância está associada a uma maior vulnerabilidade aos efeitos negativos dos acontecimentos de vida negativos (e.g., Belsky \& Fearon, 2002; Howes \& Ritchie, 1999; Erickson, Sroufe \& Egeland, 1985; Lewis, Feiring, McGuffog \& Jaskir, 1984; Rutter, 1983). No entanto, só um estudo longitudinal, que acompanhe os bebés, filhos de mães adolescentes, ao longo do tempo, poderá traçar e fornecer um quadro compreensivo das sequelas da organização de vinculação insegura na infância.

Contudo, não obstante os resultados da nossa investigação empírica, não podemos deixar de salientar que, de acordo com o modelo de trajectó- 
rias de desenvolvimento de Bowlby (1973), não se assume uma perspectiva determinista dos efeitos da qualidade da vinculação na infância. Neste modelo, as diferenças individuais não são vistas nem como características inexoravelmente manifestadas ao longo do tempo, nem como infinitamente maleáveis e facilmente alteráveis por cada nova experiência. Deste modo, podemos conceber que o espaço para a mudança é possível, dado que, numa perspectiva desenvolvimental das diferenças individuais na vinculação, o desenvolvimento subsequente do indivíduo deve ser concebido como o produto conjunto da adaptação ou história prévia e das circunstâncias actuais (Weinfield et al., 1999).

\section{Transmissão intergeracional da vinculação}

Um dos objectivos do nosso estudo foi analisar a transmissão intergeracional da vinculação, numa amostra de adolescentes e seus filhos. Para tal, a avaliação da organização da vinculação das mães adolescentes foi avaliada durante a gravidez, cumprindo, deste modo, a exigência metodológica, de acordo com a hipótese teórica de Bowlby, de que a avaliação da representação da vinculação da mãe seja efectuada antes do nascimento do filho(a), no sentido de controlar a influência das experiências com o(a) filho(a) na representação da vinculação da mãe, permitindo-nos falar, de facto, em transmissão intergeracional e não apenas em concordância intergeracional da vinculação (Fonagy et al., 1991; Ricks, 1985; Soares, 1996). Neste sentido, compararemos os nossos resultados apenas com as pesquisas que também avaliaram a representação da vinculação da mãe durante a gravidez, com base na $A A I$, e a organização comportamental da vinculação dos bebés na Situação Estranha. Assim, enquanto Fonagy e colaboradores (1991) relataram 66\% de acordo entre a classificação das grávidas adultas e dos filhos, ao nível dos três padrões de vinculação, e de $75 \%$ na dimensão de segurança versus insegurança, numa amostra de nível socioeconómico médio/elevado, Benoit e Parker (1994), numa amostra semelhante em termos socioeconómicos à de Fonagy e colaboradores, relataram $68 \%$ e $81 \%$ de acordo, ao nível dos quatro e três padrões de vinculação, Ward e Carlson (1995) reportaram 68\% de acordo, ao nível dos quatro padrões de vinculação $^{8}$, e $78 \%$ na dimensão de segurança versus insegurança com adolescentes, na nossa pesquisa obtivemos valores inferiores de acordo, de $47,5 \%$ nos três padrões de vinculação e de $55 \%$ em termos da segurança versus insegurança da vinculação ${ }^{9}$. Em comparação com estes estudos, o

\footnotetext{
${ }^{8}$ Foram incluídos o estatuto $\mathrm{U}$ das grávidas e a categoria $\mathrm{D}$ nos bebés nesta pesquisa.

${ }^{9}$ Saliente-se as diferenças, ao nível da dimensão da amostra, entre a nossa pesquisa $(\mathrm{N}=40)$ e pesquisas de Fonagy e colaboradores (1991), de Benoit e Parker (1994) e de
} 
padrão de transmissão, na nossa pesquisa, não foi tão claro. Não foi observada uma associação significativa entre as classificações na $A A I$ e na $S i$ tuação Estranha.

Numa análise mais específica dos nossos resultados, verificámos uma maior contribuição da segurança no sentido da concordância intergeracional - $64,7 \%$ das grávidas seguras, com filhos seguros, e $47,8 \%$ das grávidas inseguras, com filhos inseguros. Numa leitura mais detalhada das não concordâncias, verificámos que o maior número de desacordos se verificou nas grávidas classificadas como preocupadas (Padrão E), com bebés classificados como inseguros ambivalentes-resistentes (Padrão C) (das quais apenas 28,6\% tiveram filhos resistentes), resultado similar ao obtido por Fonagy e colaboradores (1991) e por Ward e Carlson (1995). Analisando este grupo em que se verificou o maior desacordo, observa-se que, no nosso estudo, assim como no de Ward e Carlson (1995), os bebés no Padrão C são sempre filhos de grávidas inseguras.

Um resultado desafiante, do ponto de vista conceptual, é que, das 23 grávidas inseguras, mais de metade $(\mathrm{N}=12,52,2 \%)$ vieram a ter filhos avaliados como seguros. A segurança do bebé é reportada a uma figura - é seguro ou inseguro com a mãe. Contudo, o processo de construção dessa segurança no bebé ocorreu num contexto relacional mais vasto, onde está embebida essa matriz diádica. Neste sentido, e atendendo à especificidade do contexto relacional que é preenchido por mais figuras, que, numa base regular, prestam cuidados à criança (a avó), consideramos que estes processos poderão influenciar a qualidade da vinculação do bebé à mãe.

Neste sentido, uma possível interpretação para estes casos de não concordância remete para a importância que outras figuras prestadoras de cuidados, em particular a avó, poderão ter na organização da vinculação destes bebés e, especificamente, na qualidade da vinculação à mãe, ou seja, na matriz relacional. No plano conceptual, assume-se que a vinculação se constrói a partir dos primeiros tempos de vida, no contexto de uma relação privilegiada com a(s) figura(s) que proporcionam segurança e confiança, através dos comportamentos de cuidados (Soares, 1996a). Por outras palavras, se a qualidade da vinculação está ancorada na qualidade dos cuidados, poderemos interrogar-nos se, para alguns dos bebés do nosso estudo, as avós não poderão ter-se constituído como uma base segura, influenciando o modo como os bebés organizam a sua relação de vinculação com a mãe. Nesse sentido, um desafio empírico futuro, que este estudo coloca, é procurar especificar, de forma mais detalhada, o impacto de múltiplas figuras prestadoras de cuidados ao bebé, no desenvolvimento da relação de

Ward e Carlson (1995), nas quais o número de díades é superior ao nosso $(\mathrm{N}=96$, $\mathrm{N}=77$ e $\mathrm{N}=74$, respectivamente). 
vinculação entre o bebé e a mãe, no contexto da maternidade na adolescência. Um estudo que integre a avaliação da organização da vinculação dos bebés com as avós, mas também a própria organização da vinculação destas poderá elucidar as hipóteses enunciadas, contribuindo para alargar a nossa compreensão acerca das trajectórias de desenvolvimento das relações de vinculação na maternidade na adolescência, bem como para a transmissão intergeracional dos comportamentos de cuidados.

Um outro resultado desafiante, do ponto de vista teórico, que importa discutir, agora no sentido inadaptativo, diz respeito aos seis bebés inseguros (na totalidade inseguros-evitantes) cujas mães foram avaliadas como seguras durante a gravidez. Uma vez mais, múltiplas sinergias poderão estar aqui a operar. Se a organização da vinculação das mães se tiver mantido estável ao longo do primeiro ano de vida do bebé, que seria traduzida, novamente, na não concordância mãe-filho aos 12 meses, poderemos colocar a hipótese da influências de outras figuras que poderão estar aqui a intervir. Será que, nestes casos, a prestação de cuidados ao bebé por outras figuras, nomeadamente a avó e o pai do bebé, não poderá estar a contribuir para a não concordância no sentido inadaptativo? Por exemplo, no estudo de Sagi e colaboradores (1997) em kibbutzim israelitas foram evidenciadas "limitações contextuais ou ecológicas", em termos da concordância intergeracional da vinculação mãe-filho, ao mostrarem que esta estava dependente da condição dos bebés dormirem na sua própria casa ou em grupos comuns. Só um estudo futuro poderá clarificar esta hipótese.

Uma outra leitura deste resultado é igualmente possível, no sentido de uma desarmonia na transição de papéis. As adolescentes deste estudo vivenciaram uma dupla transição, uma transição de papéis acelerada: a transição para a maternidade e, muitas vezes, a transição para a conjugalidade. Poderemos pensar se, para algumas destas adolescentes, o exercício do papel parental e a experiência real com o bebé não poderão ter sido vividos com dificuldade, ao longo do primeiro ano de vida, resultando numa prestação de cuidados ao bebé (sobretudo em situações em que este se sente ameaçado e perturbado) rejeitante das suas necessidades de vinculação e, como tal, promotora da construção de uma organização de vinculação insegura? Estes bebés que, na sua totalidade, são inseguros-evitantes parecem ter construído um modelo da figura materna como alguém indisponível, tendo, por isso, aprendido a evitar a procura de proximidade e contacto com esta, quando o sistema de vinculação está activado, minimizando as suas necessidades de vinculação, o que, segundo Main (1981), lhes oferece a garantia da manutenção da relação de vinculação.

Por outro lado, qual será o papel da vivência da conjugalidade? Os nossos resultados mostraram que a totalidade destas adolescentes seguras com filhos inseguros estava casada ou vivia em regime de união de facto, 
no $12 .^{\circ}$ mês do pós-parto. Como argumentaram Crowell e colaboradores (2002), a conjugalidade pode oferecer um distanciamento físico e psicológico das figuras de vinculação, que poderá promover mudanças nas representações das experiências de vinculação prévias. Podemos questionar-nos até que ponto esta transição para a vida conjugal vai desafiar o modo como a adolescente e o parceiro vão funcionar, como figura de vinculação um para o outro. Embora estas adolescentes percepcionassem a qualidade da relação com o pai do bebé como positiva, até que ponto é que este vai funcionar como figura de vinculação para a adolescente? Por outro lado, até que ponto é que, com a construção da intimidade conjugal e da manutenção da relação com o parceiro e das suas próprias necessidades de vinculação, algumas destas adolescentes não poderão estar a relegar para segundo plano as próprias necessidades de vinculação do filho?

Na perspectiva desenvolvimental (Sroufe, 1996), o desenvolvimento pode ser concebido como um contexto. Neste sentido, importa considerar o impacto do processo de desenvolvimento das próprias mães adolescentes na transgeracionalidade da vinculação. De acordo com Bowlby (1988), uma das características dos modelos internos dinâmicos é a possibilidade de transformação e adaptação aos novos períodos desenvolvimentais e aos contextos, ao longo do desenvolvimento do indivíduo. Para além da actualização contínua, em resposta aos novos períodos desenvolvimentais, os modelos internos dinâmicos, segundo o autor, poderão sofrer mudanças mais acentuadas, face a determinados acontecimentos, como o nascimento de um filho ou a transição para a conjugalidade. Segundo Fonagy e colaboradores (1991), a transição para a maternidade, ou seja, o exercício da maternidade, na relação efectiva com a criança, pode implicar uma mudança nos modelos internos dinâmicos. Como argumentaram Solomon e George (1996), discutindo a questão da organização da vinculação e da sua relação com o sistema de prestação de cuidados, para as mães que, na gravidez, não têm modelo interno seguro face às experiências com as suas figuras de vinculação na infância (e que não são capazes de ter uma representação dos cuidados que seja autónoma em relação ao passado), as experiências positivas no exercício do papel de figura prestadora de cuidados poderão levar a uma revisão dos seus modelos mais precoces, no sentido da segurança, e ao desenvolvimento de uma vinculação segura no filho. O exercício da maternidade vai mudar o foco das mães adolescentes, da perspectiva da infância - ser a figura vinculada - para a perspectiva de ser figura prestadora de cuidados - e, como tal, tornar-se figura de vinculação para o bebé. Neste sentido, será que a própria relação com o bebé, (co-)construída ao longo do primeiro ano de vida, não poderá ter imprimido variabilidade na trajectória de desenvolvimento de algumas destas adolescentes, e ter levado a uma alteração no sentido da segurança, em termos da organização de 
vinculação em algumas destas mães? Só um estudo que, no seu desenho metodológico, integre uma reavaliação das mães adolescentes com base na $A A I$, aos 12 meses de idade do filho, poderá ajudar a encontrar respostas para estas questões.

Por outro lado, no nosso estudo, muitas destas adolescentes, para além da transição para a maternidade, vivenciaram também a transição para uma vida conjugal; as adolescentes, em geral, avaliaram de forma positiva a relação com o parceiro/cônjuge. Neste sentido, não poderemos excluir a possibilidade de a relação continuada mantida com o companheiro, ao longo do primeiro ano de vida do bebé, de acordo com a Teoria da Vinculação, se ter constituído como uma experiência emocional significativa, servindo de "base segura" para algumas destas adolescentes e proporcionando, dessa forma, condições facilitadoras para a revisão da organização interna das relações de vinculação construídas mais precocemente (Soares, 1996a; Main \& Goldwyn, 1984-1998). Como salientaram, recentemente, Crowell e colaboradores (2002), embora discutindo questões da idade adulta, na perspectiva da vinculação, a díade conjugal cria um novo ambiente de prestação de cuidados e apresenta experiências de vinculação que podem desafiar concepções prévias, em termos de organização da vinculação. Por outro lado, há também a considerar que a própria avaliação da qualidade da organização da vinculação do pai do bebé poderia igualmente trazer alguma luz para a compreensão das origens dos casos de não concordância na vinculação mãe-bebé.

Por último, não queríamos deixar de referir que tem sido argumentado, embora em termos intra-individuais, que a discordância verificada entre a classificação na $A A I$ e na Situação Estranha pode ser explicada metodologicamente (Zimmmerman \& Becker-Stoll, 2002). Tem sido sugerido que, enquanto a $A A I$ avalia a representação actual, no presente, durante a entrevista das experiências passadas na infância (nível avaliativo-declarativo), a Situação Estranha avalia estratégias interactivas de regulação emocional que podem ser observadas como padrões comportamentais (nível procedimental), no final do primeiro ano de vida.

Em síntese, na globalidade, os resultados encontrados revelam que o modo como as mães adolescentes organizaram as suas experiências de vinculação na gravidez não está relacionado com o modo como os bebés organizam as suas relações de vinculação com elas, aos 12 meses de idade.

O nosso objectivo foi examinar o conceito de transmissão da vinculação, aquilo que é transmitido da grávida para o bebé, sem a influência da relação com o bebé, na sua organização da vinculação. Contudo, quando nos focalizamos no bebé, quando analisamos o movimento do bebé para a mãe adolescente, temos de ter presente que se tratam de dois momentos distintos: assim, enquanto a organização da vinculação da adolescente, tal 
como foi avaliada neste estudo, é independente da do bebé, a avaliação da organização do bebé pode não ser independente da da mãe, aos 12 meses de idade. Esta questão só poderia ser esclarecida com a repetição da $A A I$, aos 12 meses de idade. A não transmissão da vinculação mostra que o fenómeno não é linear. E, por isso, fará sentido falar na dialéctica do fenómeno de se transformar e ser transformado, ao longo do desenvolvimento.

"As águas onde a vinculação emerge são as águas dos cuidados" - é no exercício da função de prestação de cuidados, através do modo como a mãe se vai ajustando ao exercício da maternidade, sobretudo nas situações relevantes para a vinculação, que a qualidade da vinculação do bebé se vai construindo. Falar de transgeracionalidade supõe a existência de correntes ou de movimentos nos dois sentidos, entre a mãe e o bebé. A qualidade deste processo transaccional pode ter influência na qualidade da organização da vinculação da mãe (Jongenelen, 2004). Em síntese, múltiplas sinergias poderão contribuir para a diversidade de cenários de histórias de transgeracionalidade da vinculação.

\section{Referências}

Ainsworth, M., \& Wittig, B. (1969). Attachment and exploratory behavior of one-year-olds in a strange situation. In B. M. Foss (Ed.), Determinants of infant behavior (pp. 111-136). London: Methuen.

Ainsworth, M., \& Bell, S. (1970). Attachment, exploration, and separation: illustrated by the behavior of one-year-olds in a strange situation. Child Development, 41, 49-67.

Ainsworth, M., Blehar, M., Waters, E., \& Wall, S. (1978). Patterns of attachment: a psychological study of the strange situation. Hillsdale: Erlbaum.

Allen, J., Hauser, S., \& Borman-Spurrell, E. (1996). Attachment theory as a framework for understanding sequelae of severe adolescent psychopathology: an 11-year follow-up study. Journal of Consulting and Clinical Psychology, 64(2), 254-263.

Ammaniti, M., Speranza, A. M., \& Candelori, C. (1996). Stability of attachment in children and intergerational transmission of attachment. Psychiatria dell'Infanzia e dell'Adolscenza, 63, 313-332.

Andreozzi, L., Flanagan, P., Seifer, R., Brunner, S., \& Lester, B. (2002). Attachment classifications among 18-month-old children of adolescent mothers. Archives of Pediatrics \& Adolescent Medicine, 156, 20-26.

Belsky, J., \& Fearon, R. M. P. (2002). Infant-mother attachment security, contextual risk, and early development: a moderational analysis. Development and Psychopathology, 14, 293-310.

Benoit, D., \& Parker, K. (1994). Stability and transmission of attachment among three generations. Infant Mental Health Journal, 10, 185-202. 
Bowlby, J. (1969-1984). Attachment and loss: Vol. 1: Attachment. London: Basic Books.

Bowlby, J. (1973). Attachment and loss: Separation (Edição revista em 1985). London: Basic Books.

Bowlby, J. (1979). The making and breaking of affectional bonds. London: Tavistock.

Bowlby, J. (1980). Attachment and loss: loss, sadness and depression. London: Basic Books.

Bowlby, J. (1988). A secure base: parent-child attachment and healthy human development. London: Basic Books.

Broussard, E. R. (1995). Infant attachment in a sample of adolescent mothers. Child Psychiatry and Human Development, 25(4), 211-219.

Carlson, E. (1990). Individual differences in quality of attachment organization of high-risk adolescent mothers. Dissertação de Doutoramento não publicada, Columbia University.

Cole-Detke, H., \& Kobak, R. (1996). Attachment processes in eating disorder and depression. Journal of Consulting and Clinical Psychology, 64, 282-290.

Crowell, J. A., Treboux, D., \& Waters, E. (2002). Stability of attachment representations: the transition to marriage. Developmental Psychology, 38(4), 467-479.

Erickson, M. F., Sroufe, L. A., \& Egeland, B. (1985). The relation between quality of attachment and behavior problems in preschool in a high risk sample. In I. Bretherton \& E. Waters (Eds.), Growing points of attachment: theory and research. Monographs of the Society for Research in Child Development, 50 (1-2, Serial N. ${ }^{\circ}$ 209), 147-166.

Fonagy, P., Steele, H., \& Steele, M. (1991). Maternal representations of attachment during pregnancy predict the organization of infant-mother attachment at one year of age. Child Development, 62, 891-905.

Frodi, A., Keller, B., Foye, H., Liptak, G., Bridges, L., Grolnick, W., Berko, J., McAnarney, E., \& Lawrence, R. (1984). Determinants of attachment and mastery motivation in infants born to adolescent mothers. Infant Mental Health Journal, 5(1), 15-23.

Frodi, A., Grolnick, W., Bridges, L., \& Berko, J. (1990). Infants of adolescent and adult mothers: two indices of socioemotional development. Adolescence, 25(98), 363-374.

George, C., Kaplan, N., \& Main, M. (1984-1988). Adult Attachment Interview Protocol. Manuscrito não publicado, University of California at Berkeley.

Grossmann, K. E., Fremmer-Bombik, E., Rudolph, J., \& Grossmann, K. (1988). Maternal attachment representations as related to patterns of infant-mother attachment and maternal care during the first year. In R. A. Hinde \& J. Stevenson-Hinde (Eds.), Relationships within families: mutual influences (pp. 241-260). Oxford: Clarendon Press.

Hann, D. M., Osofsky, J. D., Stringer, S. S., \& Carter, S. S. (1988). Affective contribution of adolescent mothers and infants to the quality of attachment. Comunicação apresentada na International Conference on Infant Studies. Washington, DC. 
Hesse, E. (1999). The Adult Attachment Interview. Historical and current perspectives. In J. Cassidy \& P. S. Shaver (Eds.), Handbook of attachment: theory, research and clinical applications (pp.395-433). New York: The Guilford Press.

Howes, C., \& Ritchie, S. (1999). Attachment organizations in children with difficult life circumstances. Development and Psychopathology, 11, 251-268.

Jongenelen, I. (1998). Gravidez na adolescência: uniformidade e diversidade no desenvolvimento. Dissertação de Mestrado apresentada à Faculdade de Psicologia e de Ciências da Educação da Universidade do Porto.

Jongenelen, I. (2004). Vinculação em mães adolescentes e seus bebés - da matriz relacional à matriz contextual. Dissertação de Doutoramento apresentada ao Instituto de Educação e Psicologia da Universidade do Minho.

Kobak, R., Cole, H., Ferenz-Gillies, R., Fleming, W., \& Gamble, W. (1993). Attachment and emotional regulation during mother-teen problem solving. A control theory analysis. Child Development, 64, 231-245.

Kobak, R. (1993). The Attachment Q-sort. Manuscrito não publicado, University of Delaware.

Lamb, M. E., Hopps, K., \& Elster, A. B. (1987). Strange situation behavior of infants with adolescent mothers. Infant Behavior and Development, 10, 39-48.

Landy, S., Montgomery, J. S., Schubert, J., Cleland, J. F., \& Clark, C. (1983). Mother-infant interaction of teenage mothers and the effect of experience in the observational sessions on the development of their infants. Early Child Development and Care, 10, 165-186.

Levine, L. V., \& Tuber, S. B. (1991). Mothers' mental representations and their relationship to mother-infant attachment. Bulletin of the Menninger Clinic, 55(4), 454-470.

Lewis, M., Feiring, C., \& Rosenthal, S. (2000). Attachment over time. Child Development, 71(3), 707-720.

Main, M. (1981). Avoidance in the service of attachment: a working paper. In K. Immelmann, G. Barlow, L. Petrinovich \& M. Main (Eds.), Behavioral development: the Bielefeld interdisciplinary project. New York: Cambridge University Press.

Main, M., Kaplan, N., \& Cassidy, J. (1985). Security in infancy, childhood, and adulthood: a move to the level of representation. In I. Bretherton \& E. Waters (Eds.), Growing points of attachment theory and research. Monographs of the Society for Research in Child Development, 50 (1-2, Serial N. ${ }^{2} 209$ ), 66-104.

Main, M. (1996). Introduction to the special section on attachment and psychopathology: 2. Overview of the field of attachment. Journal of Consulting and Clinical Psychology, 64(2), 237-243.

Matos, R. (2000). Maus tratos na infância: impacto na representação da vinculação e na qualidade da interacção mãe-bebé, em mães adolescentes. Dissertação de Mestrado apresentada ao Instituto de Educação e Psicologia da Universidade do Minho. 
Pederson, D. R., Gleason, K. E., Moran, G., \& Bento, S. (1998). Maternal attachment representations, maternal sensitivity, and the infant-mother attachment relationship. Developmental Psychology, 34(5), 925-933.

Radojevic, M. (1994). Mental representations of attachment among prospective Australian fathers. Australian and New Zealand Journal of Psychiatry, 28, 505-511.

Rellinger, E. A. (1995). Percursors and consequences of insecure attachment in children of adolescent mothers. Dissertação de Doutoramento não publicada.

Ricks, M. H. (1985). The social transmission of parental behavior: attachment across generations. In I. Bretherton \& E. Waters (Eds.), Growing points of attachment theory and research (pp. 211-227).

Rutter, M. (1983). Stress, coping and development: some issues and some questions. In N. Garmezy \& M. Rutter (Eds.), Stress, coping and development in children (pp. 1-41). New York: McGraw-Hill.

Sagi, A., van IJzendoorn, M. H., Scharf, M. H., Joels, T., Koren-Karie, N., Mayseless, O., \& Aviezer, O. (1997). Ecological constraints for intergenerational transmission of attachment. International Journal of Behavioral Development, 20, 287-299.

Silva, A. P. (1998). Vinculação e funcionamento cognitivo na criança: diferenças e variabilidade no contexto e interacção mãe-filho. Dissertação de Mestrado apresentada à Faculdade de Psicologia e de Ciências da Educação da Universidade do Porto.

Soares, I. (1992). Representação da vinculação na idade adulta e na adolescência. Estudo intergeracional: mãe-filho(a). Dissertação de Doutoramento apresentada à Faculdade de Psicologia e de Ciências da Educação da Universidade do Porto.

Soares, I., Lopes dos Santos, P., Jongenelen, I., Henriques, M. R., Silva, A. P., Figueiredo, B., Mascarenhas, C., Machado, G., Neves, L., Serra, M., Silva, M. C., Cunha, J. P., \& Costa, O. (1995). Avaliação da vinculação e da regulação autonómica da frequência cardíaca na situação estranha. Vol. IV. In L. S. Almeida, S. Araújo, M. M. Gonçalves, C. Machado \& M. R. Simões (Eds.), Avaliação psicológica: formas e contextos (pp. 247-258). Braga: APPORT.

Soares, I. (1996). Representação da vinculação na idade adulta e na adolescência. Estudo intergeracional: mãe-filho(a). Braga: Centro de Estudos em Educação e Psicologia, Instituto de Educação e Psicologia, Universidade do Minho.

Soares, I. (2001). A adolescência como um tempo de (re)construção de vínculos. Comunicação apresentada no Encontro sobre Desenvolvimento Psicológico e Contexto Escolar, Barcelos.

Soares, I. (2002). A vinculação vinculada. Lição de Síntese para efeitos de Provas de Agregação, Instituto de Educação e Psicologia da Universidade do Minho.

Solomon, J., \& George, C. (1996). Defining the caregiving system: toward a theory of caregiving. Infant Mental Health Journal, 17, 183-197.

Spencer, M. S. (1997). Behavior problems in children of adolescent mothers: exploring the role of attachment as a protective factor. Dissertação de Doutoramento não publicada. 
Sroufe, L. A. (1996). Emotional development. Cambridge, England: Cambridge University Press.

Steele, H., Steele, M., \& Fonagy, P. (1996). Associations among attachment classifications of mothers, fathers and their infants. Child Development, 67, 541-555.

Thompson, R. A. (1999). Early attachment and later development. In J. Cassidy \& P. S. Shaver (Eds.), Handbook of attachment: theory, research, and clinical implications (pp. 265-286). New York: The Guilford Press.

van IJzendoorn, M., \& Kroonenberg, P. (1988). Cross-cultural patterns of attachment: a meta-analysis of the strange situation. Child Development, 59, 147-156 .

van IJzendoorn, M. (1995). Intergenerational transmission of parenting: a review of studies in nonclinical populations. Developmental Review, 12, 76-99.

van IJzendoorn, M., \& Bakermans-Kranenburg, M. (1996). Attachment representations in mothers, fathers, adolescents, and clinical groups: a meta-analytic search for normative data. Journal of Consulting and Clinical Psychology, 64, $8-21$.

van IJzendoorn, M. H. (1992). Intergenerational transmission of parenting: a review of studies in nonclinical populations. Developmental Review, 12, 76-99.

van IJzendoorn, M. H. (1995). Adult attachment representations, parental responsiveness, and infant attachment: a meta-analysis on the predictive validity of the Adult Attachment Interview. Psychological Bulletin, 117, 387-403.

van IJzendoorn, M. H. (1996). Commentary. Human Development, 39, 224-231.

Ward, M., \& Carlson, E. (1995). Associations among adult attachment representations, maternal sensivity, and infant-mother attachment in a sample of adolescent mothers. Child Development, 66(1), 69-79.

Weinfield, N., Sroufe, A., \& Egeland, B. (2000). Attachment from in infancy to early adulthood in a high-risk sample: continuity, discontinuity, and their correlates. Child Development, 71(3), 695-702.

Weinfield, N. S., Sroufe, L. A., Egeland, B., \& Carlson, E. A. (1999). The nature of individual differences in infant-caregiver attachment. In J. Cassidy \& P. S. Shaver (Eds.), Handbook of attachment: theory, research, and clinical applications (pp. 68-88). New York: The Guilford Press.

Whitman, T. L., Borkowski, J. G., Keogh, D. A., \& Weed, K. (2001). Interwoken lives: adolescent mothers and their children. New Jersey: Lawrence Erlbaum Publishers.

Zimmermann, P., Fremmer-Bombik, E., Spangler, G., \& Grossmann, K. E. (1997). Attachment in adolescence: a longitudinal perspective. In W. Koops, J. B. Hoeksma \& D. C. van den Boom (Eds.), Development of interaction and attachment: traditional and non-traditional approaches (pp. 281-292). Amsterdam: North Holland. 